\title{
Software de gestión para pedigrí y producción de camélidos del Nuevo Mundo: Pacokipu y Llamakipu
}

\author{
Management software for pedigree and production of New World Camelids: \\ Pacokipu and Llamakipu
}

\author{
Alan Cruz ${ }^{1,2}$, Gustavo Gutiérrez ${ }^{1}$, Alonso Burgos ${ }^{2}$, Renzo Morante ${ }^{2}$, \\ María Wurzinger ${ }^{1,3}$, Juan Pablo Gutiérrez ${ }^{4}$
}

\section{Resumen}

\begin{abstract}
Los registros genealógicos y productivos son el pilar importante dentro de los programas de mejora genética y genómica, donde la diversidad de datos que pueden colectarse debe ser sistematizada y relacionada, para llevar un análisis estadístico adecuado considerando todos los factores que influencian a las variables. Por otro lado, las propuestas actuales no contemplan el registro total de la población, tanto de registros genealógicos como productivos, además que no existe un software que permita sistematizar, ordenar y proveer información objetiva y medible en forma ordenada dentro del manejo de los camélidos domésticos. Por ello se planteó la construcción de un aplicativo informático que permita el recojo de los registros genealógicos y productivos de toda la población tanto de alpacas como de llamas dentro de los rebaños. Como resultado se
\end{abstract}

\footnotetext{
${ }^{1}$ Universidad Nacional Agraria La Molina, Lima, Perú

${ }^{2}$ Estación de Investigación Científica Pacomarca, Inca Tops S.A., Arequipa, Perú

${ }^{3}$ BOKU - University of Natural Resources and Life Sciences, Vienna, Austria

${ }^{4}$ Departamento de Producción Animal, Universidad Complutense de Madrid, España

${ }^{5}$ E-mail: alancruzc@outlook.com
}

Fuente financiera: Este trabajo fue financiado parcialmente por CONCYTEC a través del proyecto «IMAGEN - Innovaciones en la mejora genética altoandina: alpacas y llamas» (29-2019-FONDECYT$B M-I N C . I N V)$.

Recibido: 29 de diciembre de 2020

Aceptado para publicación: 10 de julio de 2021

Publicado: 24 de agosto de 2021

CLos autores. Este artículo es publicado por la Rev Inv Vet Perú de la Facultad de Medicina Veterinaria, Universidad Nacional Mayor de San Marcos. Este es un artículo de acceso abierto, distribuido bajo los términos de la licencia Creative Commons Atribución 4.0 Internacional (CC BY 4.0) [https:// creativecommons.org/licenses/by/4.0/deed.es] que permite el uso, distribución y reproducción en cualquier medio, siempre que la obra original sea debidamente citada de su fuente original 
construyó el aplicativo informático denominado «Pacokipu» y «Llamakipu» para alpacas y llamas, respectivamente, los cuales fueron construidos sobre la base de Microsoft Access, con dos lenguajes de programación, el primero con Macros de Microsoft Access y Visual Basic para Aplicativos (VBA) y el segundo con lenguaje Structured Query Language (SQL). El aplicativo utiliza tres elementos donde almacena y ordena la información de los registros en 23 tablas, 29 consultas y 93 formularios. El diseño del aplicativo informático permite un manejo amigable a cualquier centro de producción de camélidos domésticos, convirtiéndose en una herramienta para la gestión y administración de los animales dentro del rebaño y a la vez como un software para sistematizar la información colectada tanto de registros genealógicos como productivos, para ser usados dentro de los programas de mejora genética y genómica en alpacas y llamas.

Palabras clave: alpaca, programa genético, manejo de rebaños, llama, software de aplicación

\section{AbSTRaCT}

Genealogical and productive records are the most important pillars within genetic and genomic improvement programs, where the diversity of data that can be collected must be systematized and related, to carry out an adequate statistical analysis considering all the factors that influence the variables. On the other hand, the current proposals do not contemplate the total registration of the population, both genealogical and productive records. Moreover, there is no software that allows systematizing, ordering and providing objective and measurable information in an orderly manner within the management of domestic camelids. For this reason, the construction of a computer application was proposed allowing the collection of genealogical and productive records of the entire population of both alpacas and llamas within the herds. As a result, the computer application called «Pacokipu» and «Llamakipu» was developed for alpacas and llamas, respectively, and were based on Microsoft Access, with two programming languages, the first with Microsoft Access Macros and Visual Basic for Applications (VBA) and the second with Structured Query Language (SQL). The application uses three elements where it stores and orders the information of the records in 23 tables, 29 queries and 93 forms. The design of the computer application allows a friendly management of any domestic camelid production center, becoming a tool for the management and administration of the animals within the herd and at the same time as a software to systematize the information collected from both genealogical and productive records, to be used within the genetic and genomic improvement programs in alpacas and llamas.

Key words: alpaca, genetic program, herd management, llama, software application

\section{INTRODUCCIÓN}

El pionero y padre de la mejora genética animal moderna, Lush (1943), sostenía que la cría no debía basarse en el aspecto subjetivo del animal, sino en estadísticas cuantitativas y en información genética.
Sus trabajos tuvieron un gran impacto en todas las especies productivas que adoptaron el registro como un hábito de trabajo dentro de las granjas de producción. Sin embargo, los camélidos no han mejorado sus rendimientos productivos al ritmo de otras especies domésticas, siendo una de las razones la carencia en la conducción de registros 
genealógicos y productivos, como un hábito de trabajo dentro de los rebaños de camélidos domésticos (Quispe et al., 2018).

Existen propuestas de registros en camélidos domésticos que han juntado la evaluación y selección en un solo acto de manejo (DS.013-2011-AG 2011), donde el esfuerzo se ha centrado en el registro de genealogía, sesgando solo al registro de animales que por su fenotipo cumplieran ciertos parámetros morfológicos. La normativa establecida no contempla un programa integral, que pueda ser aplicable a centros productivos que aborden diferentes objetivos de producción. Consecuentemente, el progreso genético con información sesgada conduce a la elección errónea de reproductores durante la selección por no disponer de la información completa (Calsamiglia et al., 2015; MehrabaniYeganeh et al., 1999).

A diferencia de otras especies domésticas, como es el caso del vacuno lechero donde el $30 \%$ de la mejora productiva se puede atribuir al progreso genético (Shook, 2006). Los programas de mejora genética contemplan el recojo de información, de ser posible, de toda la población bajo control y de todos los efectos que pueden influir sobre el carácter, incluso con la llegada de la era genómica (VanRaden, 2008).

Los camélidos no deberían escapar a este tipo de enfoque de programas de mejora. El recojo de datos debe poder ser comparable entre sí, tanto en unidades, en métodos y entre rebaños. Esto podría ser mucho más eficaz si se dispone de un aplicativo informático que facilite la tarea de sistematizar la información colectada; entre estos se puede mencionar el PacoPro, un software privado cuya aplicabilidad se describe desde el 2009 en los trabajos publicados por la Estación de Investigación Científica de Pacomarca (Gutiérrez et al., 2009; Cruz et al., 2020b; Pinares et al., 2021). De otra parte, se ha demostrado que este tipo de aplicativos se mejoran cuando hay muchos usuarios involucrados en su uso que puedan ayudar a la retroalimentación (Rodriguez, 2014). Por ello, el presente trabajo tuvo como objetivo proponer un aplicativo informático de acceso libre que ayude en la gestión y administración de los rebaños de alpacas y llamas y, por otro lado, pueda conectar y analizar la información de las bases de datos en forma conjunta para las evaluaciones genéticas y genómicas de los camélidos del nuevo mundo.

\section{Materiales y Métodos}

El aplicativo informático fue desarrollado sobre los objetos de Microsoft Access. El aplicativo informático se construyó con dos tipos de instrucciones. El primero con Macros de Microsoft Access y Visual Basic para Aplicativos (VBA) para ingresar datos mediante formularios, cuya acción de almacenar, eliminar o editar puedan ejecutarse mediante enlaces. Este tipo de lenguaje también permite hacer consultas sencillas de las tablas. El segundo grupo de instrucciones se programó con lenguaje Structured Query Language (SQL), lenguaje que permite a Microsoft Access ejecutar el aplicativo, listar, filtrar, extraer, actualizar o eliminar datos que pertenecen a diferentes tablas de la base de datos. La construcción del aplicativo informático se centra en el recojo de tres tipos de datos: registro genealógico, registro productivo y registro complementario, cuyos registros pueden tener campos obligatorios y campos opcionales.

La primera tabla en construirse fue la tabla de usuario, asignándole los niveles de usuario y de administrador, este último con privilegios para editar el aplicativo. La siguiente fue la tabla clientes, que recopila a todos los actores que interactuarán dentro del aplicativo. Las siguientes tablas en crearse fueron las tablas majada y campaña, referidas a la formación de grupos y responsables en un periodo de tiempo. La tabla central del aplicativo esta referida al pedigrí, asignándole al arete como campo principal y obligatorio, con un formato indexado sin duplicados. 


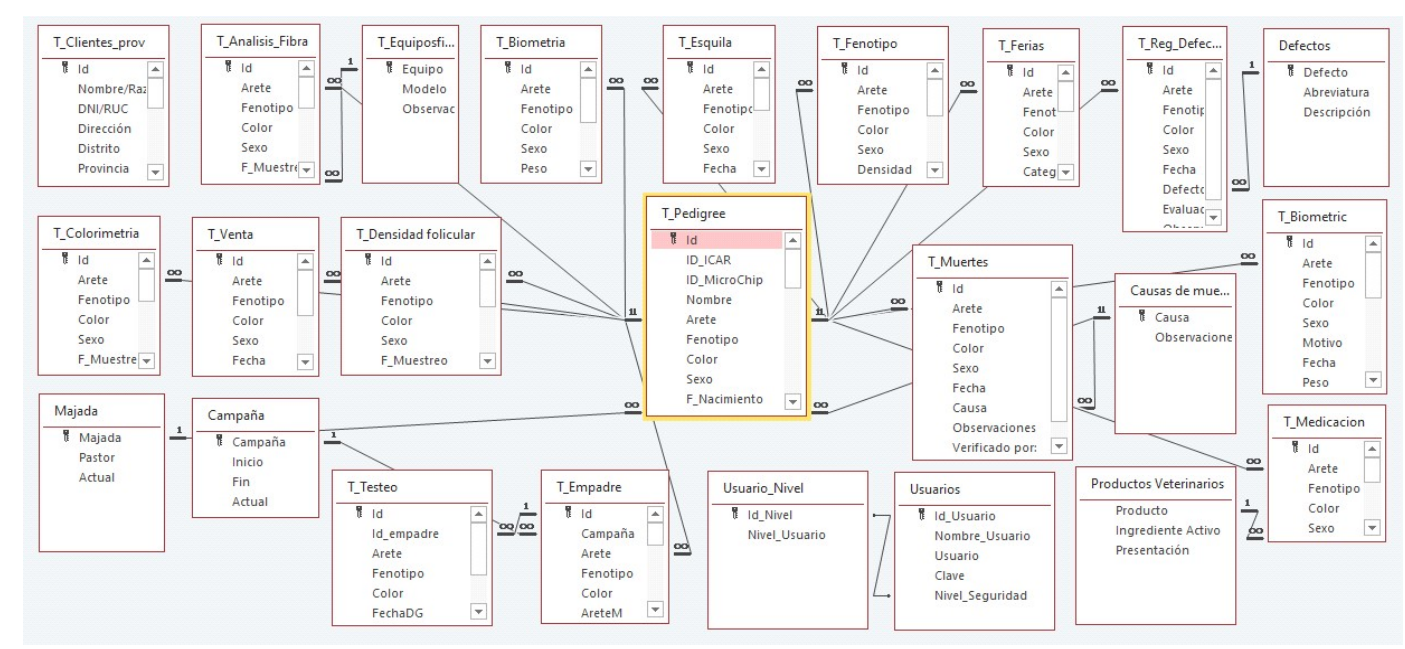

Figura 1. Relación entre tablas del software de aplicación Pacokipu y Llamakipu

Todas las demás tablas contienen una llave principal que permite ingresos repetidos del mismo animal. Todas las tablas de datos productivos están interrelacionadas al campo arete de la tabla pedigrí, para recopilar la identificación del individuo, mediante una pestaña desplegable que mitiguen errores por ingreso manual. Las relaciones entre las tablas del aplicativo se muestran en la Figura 1. La integridad referencial se puede mantener en el aplicativo mediante esta función.

\section{Registro Genealógico}

Recoge cinco campos obligatorios donde el Arete se refiere a la identificación alfanumérica que debe ser un valor único en la base de datos, en la que se sugiere combinar las iniciales del dueño con un numero correlativo, la fecha de nacimiento que corresponde a la fecha en que nació el individuo bajo el formato $\mathrm{dd} / \mathrm{mm} / \mathrm{aaaa}$, el sexo en dos niveles (macho o hembra), y Padre y Madre que corresponde a la línea directa ancestral del individuo, que cuando se desconozca a los progenitores se consignará como campo vacío. Adicionalmente, para una mejor identificación y descripción del individuo se puede utilizar el código que establece el
International Committee for Animal Recording (ICAR) que comprende 15 dígitos numéricos, de los cuales tres corresponden al código de país, tres a la institución encargada de la conducción de los registros y los otros nueve dígitos corresponden al lugar del registro y el correlativo del animal, que pueden variar según las características geopolíticas de cada país (país, región, provincia, comuna). Por otro lado, se puede utilizar el código del Microchip que establece 15 dígitos numéricos similar y concordante al código ICAR, que se establecen para cada especie, el cual debe contar con la certificación del ICAR para dispositivos de identificación por radiofrecuencia (RFID - Radio Frecuency Identification), además de la descripción del tipo, color de capa y foto del individuo, como se muestra en el Cuadro 1.

\section{Registro Productivo}

Para guardar la información del registro productivo, se diseñaron formularios con relaciones entre tablas (Figura 1), para que primero relacionen al individuo mediante el Arete desde la tabla del registro genealógico, asimismo se consigne la fecha de evaluación, el valor del fenotipo medido, y en algunos 
Cuadro 1. Campos para el registro de genealogía en el aplicativo informático

\begin{tabular}{ll}
\hline Campo obligatorio & Campo opcional \\
\hline Arete & Código ICAR ${ }^{1}$ \\
Fecha de & Código \\
nacimiento & MicroChip $^{1}$ \\
Sexo & Tipo $^{2}$ \\
Padre & Color $^{2}$ \\
Madre & Foto $^{2}$ \\
\hline${ }^{1}$ Campo opcional de identificación animal \\
${ }^{2}$ Campo opcional de descripción del individuo \\
ICAR: International Committee for Animal Recor- \\
ding
\end{tabular}

Cuadro 2. Campos para el registro productivo del aplicativo informático

\begin{tabular}{ll}
\hline Campo obligatorio & Campo opcional \\
\hline Arete & Evaluador \\
Fecha & Equipo \\
Valor del fenotipo & Observaciones \\
Motivo & \\
\hline
\end{tabular}

casos el motivo de la evaluación. Adicionalmente, los formularios pueden recoger la información del evaluador, equipo utilizado e información adicional en el campo (Cuadro 2).

Los campos de las tablas que almacenan los datos productivos tienen filtros que permiten advertir cuando los valores se encuentran fuera de rangos lógicos y diferenciar si son valores numéricos o alfabéticos. Asimismo, estas tablas tienen la posibilidad de almacenar repetibilidad en caracteres que se permita medirlo. Las unidades de peso, diámetro y longitud de fibra, volumen y ratio tienen determinadas las unidades de expresión, en tanto que los valores cualitativos presentan rangos del 1 a 5 , donde 1 representa el valor más bajo y 5 el valor deseado.

Tanto en alpacas como en llamas las tablas recogen información del análisis de fibra y medidas biométricas a cualquier edad. Asimismo, recogen información del color de la capa y de densidad folicular medida a partir de métodos no invasivos. La tabla de esquila almacena información del peso y longitud de la fibra, mientras que los registros cualitativos de eventos feriales quedarán registrados en la tabla morfología. Por otro lado, se puede registrar el sistema de reproducción al cual fueron expuestos, discriminando, por ejemplo, si se empleó inseminación artificial y transferencia de embriones; además, eventos sanitarios se almacenan en la tabla medicaciones, así como registro de defectos y diagnósticos de fertilidad.

\section{Registro Complementario}

Este tipo de registros está destinado al recojo de datos que interactúan con los registros genealógicos y productivos que no necesariamente involucran a un animal específico, sino más bien pueden incluir efectos que pueden influir la medición sobre ciertas variables. Permite discriminar características específicas de los valores de los registros, tanto genealógico como productivo, así como de los actores que se involucran dentro del manejo de camélidos. Estos registros estarán destinados a niveles de acceso en el registro del usuario, a las clases por edad en que se pueden agrupar los animales, a los tipos de taras y defectos que pueden identificarse durante el manejo, y a los grupos de pastoreo de los animales, así como a los tipos de productos que se utilizan en el manejo veterinario. Además, se puede discriminar el tipo de servicio prestan los actores que se inte- 
Cuadro 3. Tablas del aplicativo informático por tipo de registro

\begin{tabular}{lll}
\hline Registro genealógico & Registro productivo & Registro complementario \\
\hline Genealogía & Análisis de fibra & Tipo de usuario \\
& Biometría & Niveles de usuario \\
& Colorimetría & Clase de animal \\
& Densidad folicular & Tipos de defectos \\
& Reproducción & Grupos o majadas \\
& Esquila & Productos veterinarios \\
& Morfología & Clientes y proveedores \\
& Premios y ferias & Equipos \\
& Medicaciones & Causas de muertes \\
& Defectos & Destino de ventas \\
& Diagnóstico de gestación & Campaña \\
\hline
\end{tabular}

gran al aplicativo, los equipos que se utilizan en las evaluaciones de producción, las causas específicas de mortalidad y el destino que tienen los animales cuando salen del hato.

\section{Requisitos para la Instalación}

El aplicativo se ejecuta en sistemas operativos superiores de Windows XP, con una funcionalidad óptima en Windows 10, tanto de 32 y 64 bits. El aplicativo ocupa un espacio de memoria de $50 \mathrm{Mb}$, necesita una memoria mínima de RAM de 2 GB. Asimismo, necesita que el computador tenga instalado MS Office 2003 o superior, con Microsoft Excel y Access instalados. Se tiene una funcionalidad completa de los gráficos con versiones superiores a MS Office 2019.

\section{Resultados y Discusión}

Este software de aplicación para camélidos es de acceso libre y gratuito para cualquier centro de crianza y producción de camélidos, el cual se puede solicitar al correo pacokipu@pacomarca.com. El aplicativo propone la incorporación de todos los animales dentro de la construcción de la matriz de parentesco, sin discriminar las causas de descalificación, tanto en alpacas como en llamas. Asimismo, uniformiza los términos más comunes en la descripción de los individuos y propone el almacenamiento de datos en tablas independientes y relacionadas (Figura 1) mediante formularios para brindar un manejo adecuado de la información, tanto para la gestión del rebaño como para su uso en programas de mejora genética y genómica de alpacas y llamas.

El diseño del aplicativo da como resultado un sistema de registro intuitivo denominado Pacokipu para el registro de alpacas y Llamakipu para el registro de llamas, los cuales almacenan y ordenan la información de los registros en tres elementos: las tablas donde se almacena los registros, los formularios donde se ingresa y edita los registros y las consultas donde se filtra la información. 

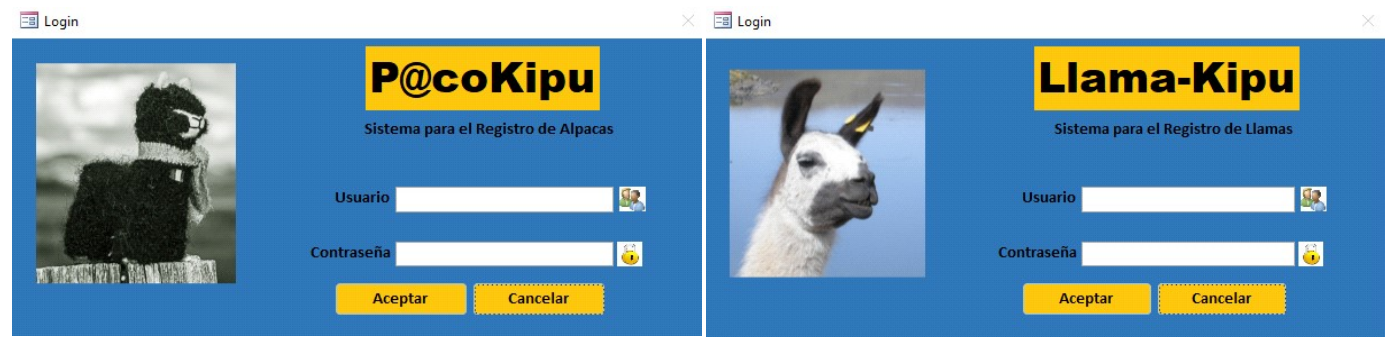

Figura 2: Interfaz gráfica de usuario de la ventana de ingreso al Pacokipu y Llamakipu

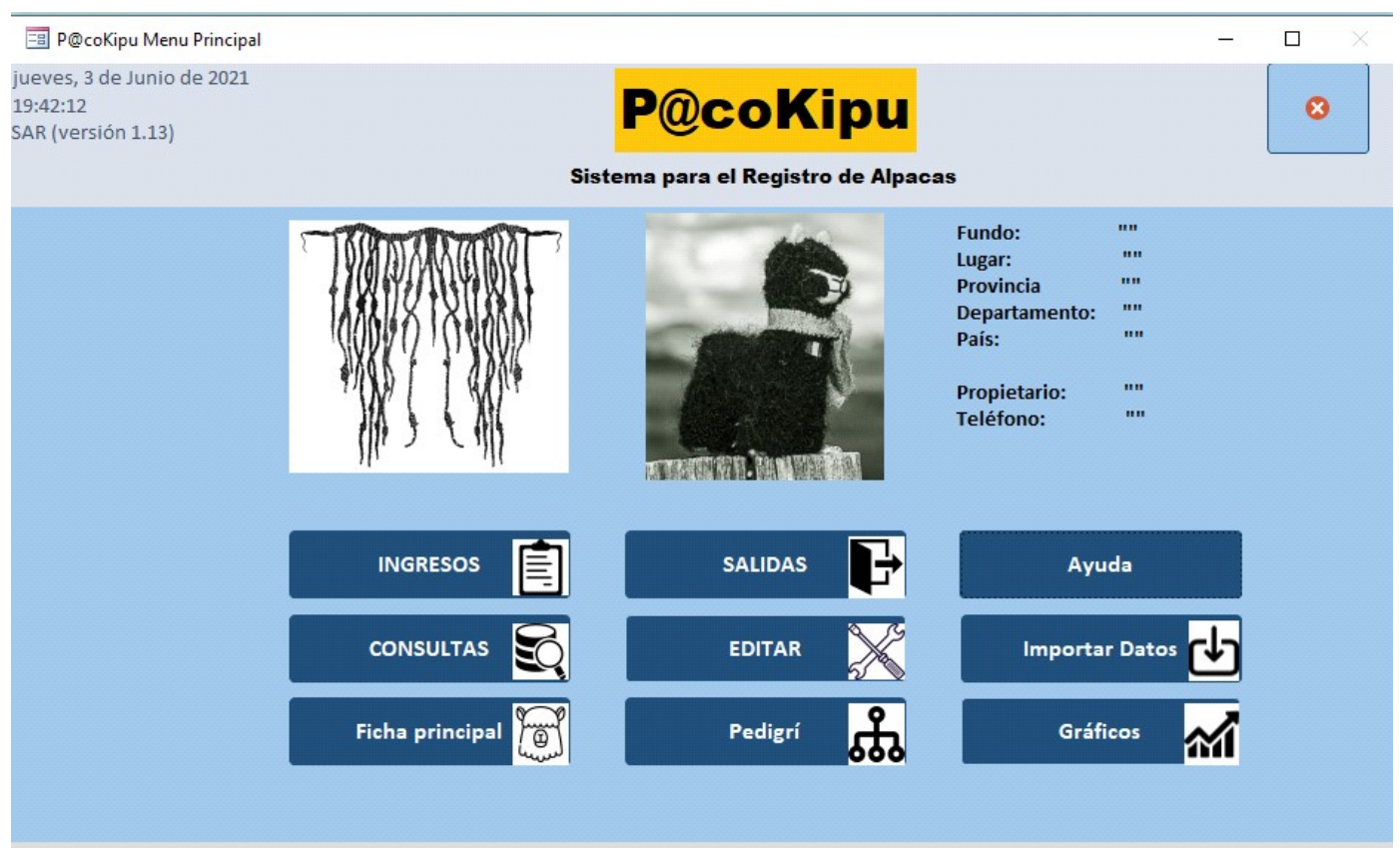

Figura 3. Interfaz gráfica de la ventana que contiene el Menú Principal del software Pacokipu

Tablas: Se han generado 23 tablas para el almacenamiento de datos (Cuadro 3), de las cuales una corresponde al registro de genealogía, 11 para los registros productivos y 11 para el registro complementario.

Consultas: Se han generado 29 tipos de consultas y referencias cruzadas, de los cuales 22 están diseñadas para la consulta entre re- gistros de genealogía y registros productivos, y siete dirigidas a los registros complementarios.

Formularios: Se han generado 93 formularios y 13 subformularios. Se tienen 31 formularios de ingreso de registros, 31 para edición de registros, 18 para listas de registros, 11 para gráficos y 2 para códigos de ejecución. 
La importancia de este aplicativo es que se propone como una herramienta diaria de la gestión de los datos y de los animales dentro del hato, con un lenguaje y formato muy amigable a cualquier centro de producción, con un enfoque de uso y utilidad práctico como se propone en este tipo de software de aplicación (Gould et al., 1991). Para la protección de la información el aplicativo cuenta con un sistema de seguridad para su acceso tanto en alpacas como en llamas (Figura 2), cuya clave y usuario permiten acceder al aplicativo identificando al usuario y el nivel de usuario.

La interfaz gráfica del software da como resultado la construcción de la primera ventana, la cual muestra información del usuario, y las acciones a seguir. Hace referencias a nueve ventanas intuitivas que guiarán al usuario (Figura 3). Estas ventanas están descritas de la siguiente manera:

- Ingresos: Aquí se permite el ingreso de los datos que interactuarán dentro del aplicativo,

- Salidas: Aquí se registran los eventos que signifiquen el movimiento de animales fuera del hato,

- Consultas: En este apartado se consulta toda la información almacenada. Tiene la opción de exportar datos a MS Excel para la creación de informes y reportes,

- Editar: En esta sección se permite la edición de errores de los registros,

- Ficha principal: Este apartado hace un reporte del animal seleccionado con información resumida de genealogía y producción. Incluye la función de crear un archivo en formato Portable Document Format (PDF),

- Pedigrí: Aquí se resume en forma gráfica el árbol genealógico del animal seleccionado,

- Gráficos: En este apartado se crean gráficos resumidos de población bajo control y de producción, con la opción de imprimir o convertir a formato PDF,
- Importar datos: Permite integrar datos grupales desde otros archivos al software de aplicación,

- Ayuda: Esta sección contiene información útil para el usuario cuando necesite ayuda o guía para el uso del software. Aquí el usuario tiene la posibilidad de desactivar y ocultar ventanas, formularios y campos del software, que no esté utilizando.

La información complementaria en la identificación individual en el registro de genealogía tiene importancia en el manejo reproductivo y análisis estadístico, porque permite la diferenciación fenotípica de alpacas (Huacaya y Suri) y llamas (Chaku y Kara). Para esa diferenciación se utilizó el concepto de tipo, por el control genético durante la segregación propuesto por Presciuttini et al. (2010) y por las características de la fibra segregada en alpacas propuesto por Pallotti et al. (2018).

Respecto a la denominación del color, si bien se pueden diferenciar hasta 22 tonalidades de colores (Morante et al., 2009), se ha demostrado que es mejor utilizar nueve tonalidades básicas para efectos de la reproducción (Cruz, 2017). Ante esto, los colores para las alpacas se han agrupado en nueve rangos: Blanco, Light Fawn intensidad X, Y, Z (LFX, LFY, LFZ), Café Claro (CC), Café Oscuro Marrón (COM), Café Oscuro Negro (CON), Negro y Gris. Para agrupar el color de las llamas se establecieron ocho rangos: Blanco, CC, COM, Negro, Gris, Aguanacado, Manchado y Conjugado. Sin embargo, se ha demostrado que el color tiene un espectro continuo, tanto en alpacas como en llamas (Cecchi et al., 2007; Cransberg et al., 2013; Cruz et al., 2021), por lo que el aplicativo informático propone además una tabla para la cuantificación objetiva del color basado en el espacio tridimensional (CIE, 2004) para las dos especies. 
Para el registro cualitativo de morfología en las dos especies se ha utilizado una escala del 1 al 5, donde 1 corresponde al valor de morfología no deseable y 5 la calificación ideal, esto como un patrón para todas las evaluaciones cualitativas en camélidos, corrigiendo las diferentes escalas que se propone en la normativa oficial (DS.013-2011AG 2011). En alpacas se proponen las evaluaciones de densidad, cabeza, calce, balance en ambos tipos y, además, el rizo para el tipo Huacaya y rulo para el tipo Suri. Este registro serviría para hacer evaluaciones cualitativas que por correlación pueden brindar ayuda en la selección como ha sido reportado por Cervantes et al. (2010). Para llamas se consideran las evaluaciones de orejas referidas a su forma, cuello referido a su grosor, cabeza referido al aspecto visual de cara, ojos y forma de la nariz; alzada referida al tamaño de la llama, largo del cuerpo, amplitud de pecho, fortaleza referida al balance de cañas y cuerpo, balance referida al aspecto general, uniformidad del color, copete referido a la cantidad de pelos sobre la frente, línea superior referido al aspecto visual del lomo, grupa referida a la ubicación y su largo respecto a la línea superior como lo reportan Machaca et al. (2020) y el DS.013-2011-AG (2011), como los principales aspectos morfológicos en llamas a ser evaluados.

Para el registro de los caracteres de fibra se tiene a la fecha de la toma de muestra como campo obligatorio. Los caracteres para registrar son: el diámetro medio $(\mu \mathrm{m})$, desviación estándar del diámetro medio $(\mu \mathrm{m})$, coeficiente de variación (\%) y el factor de confort (\%) utilizados frecuentemente como posibles criterios de selección en la mejora genética de la fibra (Cervantes et al., 2010; Cruz et al., 2020b; Gutiérrez et al., 2006). Además, en el registro se ha incluido el porcentaje de medulación (\%) y el diámetro medio de la fibra medulada $(\mu \mathrm{m})$ por su importancia para reducir el picor (Cruz et al., 2017a; Pinares et al., 2018, 2019). Adicional a esta información se registra el equipo con el cual se está haciendo el análisis, ya que existen diferentes protocolos de la norma
International Wool Textil Organization (IWTO) para los diferentes equipos y, por otro lado, el tamaño de la muestra es muy variable en los protocolos.

El registro de biometría animal recoge los pesos y tallas, donde un campo obligatorio es la fecha de la actividad para que se pueda calcular la edad del animal. Los registros en alpacas recogen las evaluaciones de peso corporal en kilogramos $(\mathrm{kg})$ y altura a la cabeza y a la cruz $(\mathrm{cm})$ como posibles criterios de selección de cara a emprender un programa cárnico o medir los efectos correlacionados con otros caracteres de interés (Cruz et al., 2017a). Para el registro de biometría en llamas, los formularios recogen los datos de peso corporal $(\mathrm{kg})$, perímetro toráxico, perímetro abdominal, altura a la cruz y largo del cuerpo $(\mathrm{cm})$ como aspectos que reflejan su relación con la producción cárnica (Machaca et al., 2020; Wurzinger et al., 2005). Además, se han considerado la altura a la grupa, altura a la cabeza, ancho de pecho, ancho de isquiones, largo de cuello, perímetro superior del cuello, perímetro inferior del cuello, largo de orejas, largo de cola y diámetro de cañas $(\mathrm{cm})$ que fueron propuestos por Machaca et al. (2020) y el DS.0132011-AG 2011. La importancia de registrar los caracteres biométricos radica en que se encuentran directamente relacionados a la producción cárnica, así como por su alta correlación con los caracteres textiles (Wurzinger et al., 2005; Cruz et al., 2017a).

El registro de la esquila es la actividad que resume la producción de fibra. El aplicativo informático ha sido diseñado para recoger la información bajo el sistema establecido por la Norma Técnica Peruana de Buenas Prácticas de Esquila y Manejo de Vellón, donde el peso del vellón se recoge en tres partes: peso del manto, peso del cuello y peso de las bragas (NTP.231.370 2014). Además, este mismo sistema de esquila se puede implementar a la esquila de llamas de tipo Chaku. Los campos obligatorios para este registro son la fecha de la esquila, pesos del vellón $(\mathrm{kg})$ y longitud de mecha $(\mathrm{cm})$, a fin de 
utilizarlos como posibles criterios de selección para incrementar el peso del vellón o incrementar el valor textil en alpacas (Gutiérrez et al., 2009). Asimismo, el recojo de estos datos permitiría en un futuro medir el crecimiento de la fibra y poder plantearse tres esquilas en los dos primeros años de vida, ya que la industria textil necesita longitudes a partir de 65 y $70 \mathrm{~mm}$ (Cruz, 2017).

El aplicativo ha propuesto una tabla para el registro de defectos, lo que permitiría identificar a animales aptos y no aptos para su reproducción y producción. Muchas veces se deja de registrar a los animales que no son aptos para la producción o que por diferentes causas no son aptos para la reproducción, ya que supone un gasto innecesario en el sistema de registro. Se ha demostrado que los programas de mejora genética avanzan cuando se utiliza toda la información de animales deseables y no deseables (MehrabaniYeganeh et al., 1999; Calsamiglia et al., 2015). Es mejor utilizar toda la información disponible haciendo un ranking y utilizar ambos extremos de la población, tanto para selección como para hacer saca dirigida (MacNeil et al., 1998; Mehrabani-Yeganeh et al., 1999). Este aplicativo informático permitiría esta adecuación para el registro y uso de la información de todos los individuos dentro del rebaño, sean alpacas o llamas, con o sin defectos genéticos, entendiendo que es mejor registrar y luego retirar el individuo del rebaño, que retirar sin registrar.

Respecto al empadre y parición en camélidos, cuya actividad se considera estacional y que para la zona andina comprende los meses de diciembre a marzo, con una fecha promedio de nacimiento para el día 26 de enero y un límite de campaña para el mes de agosto (Cruz et al., 2020a). Establecer este límite de campaña, y la campaña en sí en vez del año calendario es crucial para el manejo de los registros respecto al empadre y parición. Esta campaña permitiría agrupar individuos nacidos entre los meses de diciembre a marzo (abarcando dos años calenda- rios) en una misma campaña. Similar razonamiento es el que debe seguir el sistema de empadre. Por ello, el software de aplicación permite establecer primero la campaña antes de asignar un empadre o parición, sugiriendo el límite el mes de agosto como lo reportado por Cruz et al. (2020a). El formulario de empadre recoge la fecha de empadre, la identificación del macho y hembra, tipo de servicio (monta natural, inseminación artificial o si es destinado a la transferencia de embriones) y el tiempo de cópula. El aplicativo permite el registro de todos los eventos ocurridos durante el empadre, sin discriminar o dejar de imputar un empadre cuando el tiempo sea menor a 10 minutos como lo propone el reglamento de registros genealógicos (DS.013-2011-AG 2011), ya que se ha demostrado que en el $95 \%$ de veces el tiempo de cópula se encuentra en un rango de 4.21 a 34.78 minutos (Cruz et al., 2015).

Asimismo, el aplicativo permite el registro de los nacimientos teniendo en cuenta la fecha más probable de parición. Para esto es necesario utilizar el empadre dirigido a diferencia del empadre controlado, ya que el empadre controlado si bien permite el control de los progenitores, este no restringe el uso de que se pueda cambiar de macho dentro de la misma campaña, y esto puede conllevar a errores de imputación de padres al momento de la parición (Cruz et al., 2015; Vaughan y Tibary 2006). El empadre dirigido puede ayudar a disminuir este error de imputación, ya que se programa un macho para toda la campaña, y se puede cambiar cuando en el diagnóstico de gestación se compruebe que la fertilidad no ha sido exitosa. Por otro lado, para la construcción del árbol de pedigrí se ha utilizado las consultas recursivas en tres niveles de búsqueda, ya que se ha demostrado que es necesario controlar la consanguinidad hasta el tercer grado de parentesco, con el fin de evitar se depriman algunos caracteres (Sheikhlou et al., 2020), salvo que esta sea dirigida intencionalmente para potenciar algunas cualidades deseables por retro cruza. 
El aplicativo también recoge información objetiva respecto a la colorimetría, ya que hay un interés creciente de conservación y producción de animales por su color de capa (Cruz et al., 2021); además de un mercado que está pensando en la sostenibilidad, donde es necesario registrar la cuantificación del color de capa para ser usado dentro de los programas de producción. Este aplicativo permite recoger la fecha de la toma de muestra, así como los valores de $\mathrm{L}^{*}, \mathrm{a}^{*}, \mathrm{~b}^{*}$ del espacio tridimensional donde $\mathrm{L}^{*}$ corresponde a la Luminosidad, $\mathrm{a}^{*}$ representa la escala rojo-verde y $b^{*}$ representa la escala azul- amarillo (CIE, 2004, Cruz et al., 2021).

El software se acomoda a la nueva forma de evaluación no invasiva de densidad folicular, dejando atrás las evaluaciones por cortes histológicos y adoptando la alternativa de usar una microcámara y evaluar mediante microfotografía el número de ductos y el número de fibras por ducto, además de colectar la fecha de la evaluación y el área juzgada.

Otro tipo de registro que permite almacenar el aplicativo es la participación en eventos demostrativos o ferias. Animales ganadores en ferias pueden poseer ciertas características fenotípicas del agrado del juzgador y es posible que estas características morfológicas se transmitan a la descendencia, por lo que deben ser evaluadas y correlacionadas con la evaluación morfológica de toda la población. Por eso, el aplicativo se ha adecuado para el registro de la participación en ferias y del lugar que ocupa dentro del concurso, para que estos datos puedan integrarse dentro de las evaluaciones genéticas si fuera necesario.

Asimismo, el aplicativo permite el listado, filtrado y la interacción entre datos de diferentes tablas para poder discriminar mejor los efectos que pueden influenciar a determinado carácter. Esto permitiría tener una me- jor estimación de los componentes de varianza y una mejor precisión de la predicción del mérito genético de los caracteres que se pueden evaluar. Se reconoce el efecto de la gestación y de la lactación sobre caracteres textiles que influencian al diámetro, donde este puede variar en más de $1 \mu \mathrm{m}$ dependiendo del estado fisiológico en que la hembra se encuentre (Cruz et al., 2017b); asimismo, el efecto de la edad y el número de esquila sobre el diámetro de la fibra (Cruz et al.2020b; Gutierrez et al., 2011). De otra parte, el estado de salubridad de los animales también puede considerarse como efectos dentro de la producción, pero también pueden evaluarse como criterios de selección para tolerancia y resistencia a diferentes enfermedades y patógenos, por lo que el aplicativo ha sido diseñado para registrar los eventos de medicaciones como son la vacunación, dosificación, tratamientos, y suplementaciones.

El aplicativo informático está diseñado para llevar el control de la población cuando haya movimiento de animales (para prestar, reingresar, devolver, vender animales), haciendo resúmenes mediante gráficos del número total de la población por tipo y sexo. También se ha diseñado un formulario para que resuma las principales características de un individuo seleccionado en una ficha individual, donde esta se puede imprimir y/o convertir a formato PDF para que pueda ser compartido en formato electrónico. Asimismo, toda la información que recoge y almacena el aplicativo informático se puede exportar a Microsoft Excel.

Además de involucrar diferentes tipos de interacción entre caracteres y efectos, el aplicativo proporciona información ordenada para ser usada dentro de la misma gestión del rebaño tanto de alpacas como de llamas. Además, el usuario tendría la posibilidad de seleccionar diferentes criterios de selección dependiendo de su objetivo económico o integrarlos a través de un índice genético (Gutierrez et al., 2014), para seleccionar y reproducir alpacas y llamas. 
Los derechos de autoría del aplicativo recaen sobre todos los autores, así como las mejoras por retroalimentación. Sin embargo, la propiedad será administrada por la Universidad Nacional Agraria La Molina e Inca Tops S.A., quienes cederán este derecho, mediante un convenio de uso con los productores, quienes no podrán cederlo a terceros. La propiedad de los datos son derechos enajenables de los productores de camélidos. Sin embargo, el uso de los datos es de responsabilidad del usuario. Se sugiere realizar copias de seguridad en forma periódica para salvaguardar la base de datos. Por otro lado, es necesario integrar el software a programas de mejora de alpacas y llamas, supervisadas por un organismo externo, para garantizar la fiabilidad de la información almacenada.

\section{Conclusión}

El diseño del aplicativo informático permite un manejo amigable a cualquier centro de producción de camélidos domésticos, convirtiéndose en una herramienta para la gestión y administración de los animales dentro del rebaño, y a la vez como un software para sistematizar la información colectada tanto de registros genealógicos como productivos, para ser usados dentro de los programas de mejora genética y genómica en alpacas y llamas.

\section{Literatura Citada}

1. Calsamiglia S, Castillejos L, Astiz S, Baucelis Q. 2015. Desarrollo de un simulador de explotaciones lecheras para valorar el efecto de diferentes estrategias productivas sobre el rendimiento técnico y conómico. En: XVI Jornadas sobre Producción Animal. Zaragoza, España.

2. Cecchi T, Valbonesi A, Passamonti P, Frank E, Renieri C. 2007. Quantitative variation of melanins in llama (Lama glama L). Small Ruminant Res 71:52-
58. doi: 10.1016/j.smallrumres.2006.04.011

3. Cervantes I, Pérez-Cabal MA, Morante R, Burgos A, Salgado C, Nieto B, Goyache F, et al. 2010. Genetic parameters and relationships between fibre and type traits in two breeds of Peruvian alpacas. Small Ruminant Res 88: 6-11. doi: 10.1016/j.smallrumres.2009.10.016

4. CIE. 2004. Colorimetry. CIE 15, Technical Report. $3^{\text {rd }}$ ed. Washington DC, USA.

5. Cransberg $R$, Wakamatsu $K$, Munyard K. 2013. Melanin characterisation suggests that the «brown» phenotype in alpaca (Vicugna pacos) is predominantly pheomelanic. Small Ruminant Res 114: 240-246. doi: 10.1016/j.smallrumres.2013.07.004

6. Cruz A. 2017. Parámetros genéticos de caracteres funcionales y secundarios en alpacas. Tesis Doctoral. Madrid, España: Univ. Complutense de Madrid. 171 p.

7. Cruz A, Cervantes I, Burgos A, Morante R, Gutierrez JP. 2015. Estimation of genetic parameters for reproductive traits in alpacas. Anim Reprod Sci 163: 48-55. doi: 10.1016/j.anireprosci.2015.-09.017

8. Cruz A, Cervantes I, Burgos A, Morante R, Gutierrez JP. 2017 a. Genetic parameters estimation for preweaning traits and their relationship with reproductive, productive and morphological traits in alpaca. Animal 11: 746-754. doi: 10.1017/S175173111600210X

9. Cruz A, Gutierrez JP, Torres R, Formoso-Rafferty $N$, Morante $R$, Burgos A, Cervantes I. 2020a. Calving date and its variability as a potential trait in the breeding objective to account for reproductive seasonality in alpacas. Reprod Domest Anim 55: 814-821. doi: 10.1111/rda.13689

10. Cruz A, Menéndez-Buxadera A, Gutiérrez, G, Morante R, Burgos A, Gutiérrez JP. 2020b. Genetic (co)variance across age of fiber diameter and 
standard deviation in Huacaya alpacas, estimated by repeatability, multi-trait and random regression models. Livest Sci 231: 103863. doi: 10.1016/j.livsci.2019.103863

11. Cruz A, Morante $R$, Cervantes I, Burgos A, Gutiérrez JP. 2017b. Effect of the gestation and lactation on fiber diameter and its variability in Peruvian alpacas. Livest Sci 198: 31-36. doi: 10.1016/j.livsci.2017.02.006

12. Cruz A, Yucra A, Gutiérrez GA, Burgos A, Morante R, Gutiérrez JP, Wurzinger M. 2021. Colorimetry analysis of coat color and its relationship with fiber traits in alpacas. Animal 15: 100219. doi: 10.1016/j.animal.2021.100219

13. DS.013-2011-AG. 2011. Aprueban el reglamento de los registros genealógicos de alpacas y llamas del Perú. Ministerio de Agricultura del Perú. [Internet]. Disponible en: https://www.minagri.gob.pe/ portal/download/pdf/marcolegal/ normaslegales/decretossupremos/2011/ ds13-2011-ag.pdf

14. Gould JD, Boies SJ, Lewis C. 1991. Making usable, useful, productivityenhancing computer applications. Commun ACM 34: 74-85. doi: 10.1145/ 99977.99993

15. Gutierrez JP, Cervantes I, Perez-Cabal MA, Burgos A, Morante R. 2014. Weighting fibre and morphological traits in a genetic index for an alpaca breeding programme. Animal 8: 360-369.

16. Gutiérrez JP, Fernández I, Alvarez I, Royo LJ, Goyache F. 2006. Sire $\mathrm{x}$ contemporary group interactions for birth weight and preweaning growth traits in the asturiana de los valles beef cattle breed. Livest Sci 99: 61-68. doi: 10.1016/ j.livprodsci.2005.05.021

17. Gutiérrez JP, Goyache F, Burgos A, Cervantes I. 2009. Genetic analysis of six production traits in Peruvian alpacas. Livest Sci 123: 193-197. doi:10.1016/ j.livsci.2008.11.006
18. Gutierrez JP, Varona L, Pun A, Morante R, Burgos A, Cervantes I, Perez-Cabal MA. 2011. Genetic parameters for growth of fiber diameter in alpacas. J Anim Sci 89: 2310-2315. doi: $10.2527 /$ jas.2010-3746

19. Lush J. 1943. Animal breeding plans. Ames: Exford Press. 444 p.

20. Machaca V, Dueñas L, Bustinza V, Machaca R, Escobedo M, Quispe JE. 2020. Caracterización morfológica de las llamas (Lama glama) de la raza Ch'acu de Cusco, Perú. Rev Inv Vet Perú 31: e17821. doi: 10.15381/rivep.v31i2.17821

21. MacNeil MD, Urick JJ, Snelling WM. 1998. Comparison of selection by independent culling levels for below-average birth weight and high yearling weight with mass selection for high yearling weight in line 1 hereford cattle. J Anim Sci 76: 458-467. doi: 10.2527/ $1998.762458 \mathrm{x}$

22. Mehrabani-Yeganeh H, Gibson JP, Uimari P. 1999. The effect of using different culling regimens on genetic response with two-trait, two-stage selection in a nucleus broiler stock. Poultry Sci 78: 931-936. doi: 10.1093/ps/ 78.7.931

23. Morante R, Goyache F, Burgos A, Cervantes I, Pérez-Cabal MA, Gutiérrez JP. 2009. Genetic improvement for alpaca fibre production in the peruvian altiplano: The pacomarca experience. Anim Genet Resour Inf 45: 37-43. Doi:10.1017/S1014233909990307

24. NTP.231.370. 2014. Norma técnica peruana: 231.370-2014. Buenas prácticas de esquila y manejo de vellón. Comisión de Normalización y de Fiscalización de Barreras Comerciales no Arancelarias - INDECOPI, Perú. [Internet]. Disponible en: https://www.gob.pe/ institucion/inacal/noticias/79254-inacalaprueba-norma-tecnica-peruana-enquechua-sobre-buenas-practicas-de-esquila-y-manejo-del-vellon-de-fibra-de-alpaca 
25. Pallotti S, Pediconi D, Subramanian D, Molina MG, Antonini M, Morelli $M B$, Renieri $C$, et al. 2018. Evidence of post-transcriptional readthrough regulation in FGF5 gene of alpaca. Gene 647: 121-128. doi: 10.1016/j.gene.2018.01.006

26. Pinares $R$, Cruz A, Daverio MS, Gutiérrez JP, Ponce de León FA, Wurzinger M, Di Rocco F, Gutiérrez GA. 2021. Polimorfismos de nucleótido simple (PNSs) del gen MC1 R en alpacas negras y marrones. Rev Per Biol 28: e19742. doi: 10.15381/rpb.v28i1.19742

27. Pinares R, Gutiérrez GA, Cruz A, Morante R, Cervantes I, Burgos A, Gutiérrez JP. 2018. Heritability of individual fiber medullation in Peruvian alpacas. Small Ruminant Res 165: 93100. doi: 10.1016/j.smallrumres.2018.04.007

28. Pinares R, Gutiérrez G, Cruz A, Burgos A, Gutiérrez JP. 2019. Variabilidad fenotípica del porcentaje de fibras meduladas en el vellón de alpaca Huacaya. Rev Inv Vet Perú 30: 699-708. doi: 10.15381/rivep.v30i2.16098

29. Presciuttini S, Valbonesi A, Apaza N, Antonini M, Huanca T, Renieri C. 2010. Fleece variation in alpaca ( $\mathrm{Vicu}$ gna pacos): a two-locus model for the Suri/Huacaya phenotype. BMC Genet 11: 70. doi: 10.1186/1471-2156-11-70
30. Quispe E, Mueller JP, Bejar J, Alfonso L, Gutiérrez G 2018. Actualidades sobre adaptacion, producción, reproducción y mejora genética en camélidos. Perú: Univ. Nacional de Huancavelica.

31. Rodriguez W. 2014. Software libre para educación e investigación en ingeniería. Rev Educ Ing 9: 18-22.

32. Sheikhlou M, Badereh S, Bahri Binabaj F. 2020. Assessment of between-founder heterogeneity in inbreeding depression for reproductive traits in baluchi sheep. Anim Reprod Sci 221: 106593. doi: 10.1016/j.anireprosci.2020.-106593

33. Shook GE. 2006. Major advances in determining appropriate selection goals. J Dairy Sci 89: 1349-1361. doi: 10.3168/ jds.S0022-0302(06)72202-0

34. VanRaden PM. 2008. Efficient methods to compute genomic predictions. J Dairy Sci 91: 4414-4423. doi: 10.3168/jds.20070980

35. Vaughan JL, Tibary A. 2006. Reproduction in female South American camelids: a review and clinical observations. Small Ruminant Res 61: 259-281. doi: 10.1016/j.smallrum-res.2005.07.015

36. Wurzinger M, Delgado J, Nürnberg M, Valle Zárate A, Stemmer A, Ugarte G Sölkner J. 2005. Growth curves and genetic parameters for growth traits in Bolivian llamas. Livest Prod Sci 95: 7381. doi: 10.1016/j.livprodsci.2004.12.015 